\title{
Base Station Sleeping Mechanism for Reduced Delay Using Traffic Load Prediction
}

\author{
Ali Alnoman and Alagan Anpalagan ${ }^{+}$ \\ Ryerson University, Toronto, Canada
}

\begin{abstract}
Small cells are expected to be densely deployed in future networks to improve spectral and energy efficiencies. Due to the their small coverage and fluctuating number of users, the On-Off mechanism in small cell base stations (SBSs) has to be dynamically adapted in order to reduce the total energy consumption. However, the time delay associated with the transition from the Off to ON states can degrade the network performance. In this paper, a traffic prediction algorithm is proposed to perform a proactive SBS activation by anticipating future workload in SBS clusters using information of distance and received signal power of associated users. The distance and power measurements are smoothed using Haar wavelet filters to get a better approximation of the cluster's traffic load. Each SBS operates in a self-organized manner in coordination with the neighbouring SBSs in that cluster wherein the information of associated users are exchanged among SBSs. The work aims to adaptively modify the On-Off mechanism while minimizing the time delay that is incurred from the wake-up process of SBSs. Simulation results show that the proposed algorithm can significantly reduce the delay with a slight increase in power consumption.
\end{abstract}

Keywords: small cells, sleeping mechanism, wavelet, traffic prediction, delay, energy efficiency.

\section{Introduction}

Due to the high quality of service (QoS) requirements in future cellular networks along with the massive number of users, the dense deployment of small cells which facilitate spatial frequency reuse emerged as a promising solution to improve both network capacity and energy efficiency. The increasing energy costs and $\mathrm{CO}_{2}$ emissions make the dynamic performance of base stations (BSs), which consume about $60-80 \%$ of the network's energy, an essence in the design of cellular networks [1].

The frequent spatial and temporal variations in traffic load impose an inefficient exploitation of BS's resources and energy. Therefore, BSs have to be dynamically controlled to preserve energy [2] such that the energy consumption is adapted to the variations in traffic load [3]. Due to their small coverage area, small BSs (SBSs) are more prone to having unassociated users [4]. Moreover, femtocell BSs are underutilized most of the time because they are basically designed to provide service in full load conditions. Therefore, adopting efficient sleeping mechanisms is essential to avoid the operation of unnecessary BSs to reduce energy wastage [5] and to minimize the interference among neighboring cells [6]. Moreover, SBSs which are described as plug-and-play devices should have self-organizing features to facilitate network management.

Despite the aforementioned advantages, BS sleeping can also increase the time delay experienced by users [7]. Moreover, coverage holes, which deteriorate users' experience, might emerge as a side effect of BS sleeping [3]. To overcome this problem, users within the area of a sleeping BS can be served by neighboring BSs which increase the transmit power to compensate for the shortage in wireless coverage [8].

The on-off mechanism for improving energy efficiency has been widely studied recently. An artificial neural network (ANN)-based traffic prediction was proposed in [9] to switch-off femtocell BSs under low traffic load conditions. In [10], an optimization of resource partitioning and user association was proposed to

\footnotetext{
Corresponding author.

E-mail address: (alagan@ee.ryerson.ca).
} 
minimize the total energy consumption. The work assumed that both macro and small cell BSs are allowed to enter the sleep mode.

Several sleep depth levels were introduced in [8] to improve energy efficiency in a k-tier heterogeneous network (HetNet)f using random and strategic sleeping policies. It was shown that the energy efficiency was improved using random sleeping policy and further improved with the strategic sleeping policy. In [11], an optimal sleep/wake-up scheme was derived based on Markov decision process (MDP) using traffic load and user localization in HetNets to minimize energy consumption, while preserving the QoS experienced by users.

In this paper, the distance and received signal power of users associated with each SBS in a cluster are collected and decomposed using wavelet filters. Then, the mean values of those data are calculated. The proposed algorithm is applied to anticipate the future load in each SBS. When the load on a certain SBS is light and can be offloaded to a macro BS, the operation of that SBS is considered as unnecessary, and the SBS is switched from the On state to the standby or Off states.

The rest of the paper is organized as follows. Section 2 describes the system model. Section 3 introduces the simulation setup. Results and discussion are presented in Section 4, and finally, Section 5 provides conclusion of the work.

\section{System Model}

Base stations have three main blocks: (1) processing unit, (2) power amplifiers and radio-frequency (RF) transmitters, and (3) field-programmable gate array (FPGA). By controlling the operation of these blocks, different sleep modes can be obtained as follows [8]:

- ON: All blocks are active and maximum power is consumed.

- STANDBY (light sleep): The RF module is shutdown while other units remain operational, and hence the BS can be quickly activated.

- OFF: All blocks are shutdown, and the power consumption of the BS is considered to be zero.

Table I: Wake-Up Times and Power Consumption for the Suggested Sleeping Modes [8]

\begin{tabular}{|c|c|c|}
\hline Sleeping Mode & Sleep Mode Wake-Up Time (sec) & Power Consumption (\%) \\
\hline ON & 0 & $100 \%$ \\
\hline STANDBY & 0.5 & $50 \%$ \\
\hline OFF & 30 & $0 \%$ \\
\hline
\end{tabular}

The network is modeled using stochastic geometry which simulates the real randomness of SBSs with their associated users [1]. The SBSs and users are distributed using the hard core point process (HCPP) model in which $\mathbf{B}=\{1 \ldots b\}$ represents the set of SBSs, whereas $\mathbf{U}=\{1 \ldots u\}$ represents the set of associated users which are normally distributed around SBSs. Fig. 1 depicts a two-tier HetNet.

The network is assumed to have enough capacity, and once an SBS is Off, users are assumed to be offloaded to a macro BS that co-exists in that particular area. The path loss measured in $\mathrm{dB}$ is calculated by the indoor hotspot (InH) model from the international telecommunication union report (ITU-R) as follows:

$$
\gamma=16.9 \log _{10}(d)+32.8+20 \log _{10}\left(f_{c}\right)
$$

where $d$ represents the distance between the SBS and the associated user measured in meters, and $f_{c}$ is the carrier frequency measured in GHz. In addition, shadow fading that encounters the signal between SBSs and users is approximated statistically by the normal distribution:

$$
\mathrm{X}_{\sigma}(d B) \sim N\left(\mu, \sigma^{2}\right)
$$

where $\mu$ and $\sigma$ are the mean and standard deviation of the fading depth measured in $\mathrm{dB}$, respectively. The mobility of user locations is simulated by adjusting the standard deviation of the user distances that are normally distributed around the SBS location. Now, the distance vector $\mathbf{d}=\left\{d_{1}, d_{2}, \ldots, d_{k}\right\}$ and power vector 
$\mathbf{p}=\left\{p_{1}, p_{2}, \ldots, p_{k}\right\}$ are obtained and decomposed using the Haar wavelet filters (one-level decomposition) to obtain a smoother approximation. Afterwards, the approximated coefficient vectors $\Phi_{d}$ and $\Phi_{p}$ are considered as the distance and power data used our algorithm. The mean values of the output vectors for each SBS are calculated as follows:

$$
\begin{array}{ll}
\lambda_{d(i)}=E\left[\Phi_{d(i, j)}\right], & \forall i \in \boldsymbol{B}, \forall j \in \boldsymbol{U} \\
\lambda_{p(i)}=E\left[\Phi_{p(i, j)}\right], & \forall i \in \boldsymbol{B}, \forall j \in \boldsymbol{U}
\end{array}
$$

The operator $E($.$) is the expectation operator. The maximum value of \lambda_{d}$ and $\lambda_{p}$ for all SBSs in the cluster are then obtained:

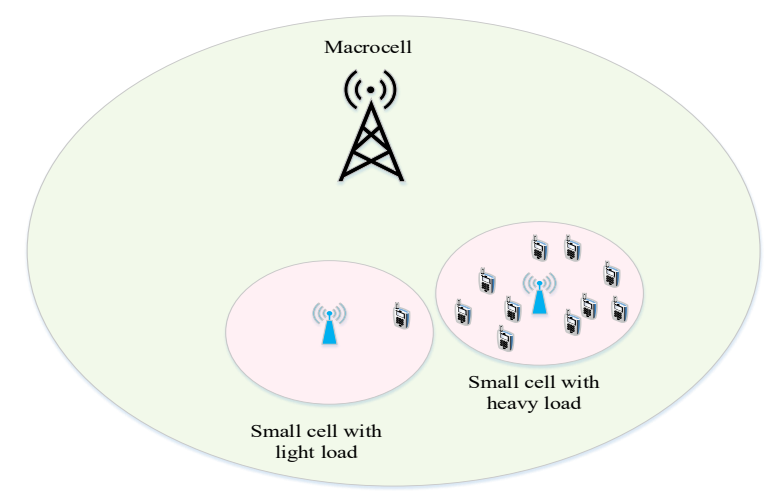

Fig. 1: A two-tier HetNet consisting of one macro cell and two small cells with different load conditions.

$$
\begin{array}{cc}
\tau_{d}=\max \left\{\lambda_{d(i)}\right\}, & \forall i \in \boldsymbol{B}, \\
\tau_{p}=\max \left\{\lambda_{p(i)}\right\}, & \forall i \in \boldsymbol{B}
\end{array}
$$

Here, $\tau_{d}$ and $\tau_{p}$ represent the threshold (maximum) distance and power, respectively. These threshold parameters indicate the highest workload level in the cluster. The time delay is calculated based on Table I. The mean delay $\Delta$ experienced by users in all SBSs is calculated as in (7).

$$
\Delta=E\left[L_{(i, j)}\right], \quad \forall i \in \boldsymbol{B}, \forall j \in \boldsymbol{U}
$$

where $L$ represents the delay experienced by a mobile user $j$ associated with an SBS $i$. This work aims at minimizing the delay of SBS activation by predicting future traffic load in a network cluster based on current traffic measurements. By avoiding the possibility of having active users in sleeping cells, the time delay can be significantly reduced. The proposed sleeping mechanism is described in Algorithm 1 (Fig. 2(a)).

In this algorithm, $U$ denotes the number of active users associated with a particular SBS, whereas $\lambda_{(i)} \in\left\{\lambda_{d(i)}, \lambda_{p(i)}\right\}$ and $\tau \in\left\{\tau_{\mathrm{d}}, \tau_{\mathrm{p}}\right\}$. When a single user $(\mathrm{U}=1)$ is associated with an SBS, and the traffic load in that SBS which is denoted by $\lambda_{d(i)}$ or $\lambda_{p(i)}$ is greater than the thresholds $\tau_{d}$ or $\tau_{p}$, this means that the entire cluster area is lightly loaded, and that SBS can be switched off without incurring significant delay. It is also assumed that when only one user is associated with an SBS, that user can be offloaded to a macro BS, and the SBS can be forced to the Standby or Off modes. Fig. 2 (b) shows the flowchart of the sleeping mechanism.

\section{Simulation Setup}

The network is modeled using stochastic geometry, namely the hard core point process, wherein 10 SBSs forms a cluster such that no two SBSs coexist within the same coverage area. The algorithm is tested using two strategies; the first deals with analyzing only the Euclidean distance between SBSs and the associated 
users, and the second considers the received signal power along with the effects of path loss and shadow fading imposed in the signal path between SBSs and users. Table II summarizes the system parameters.

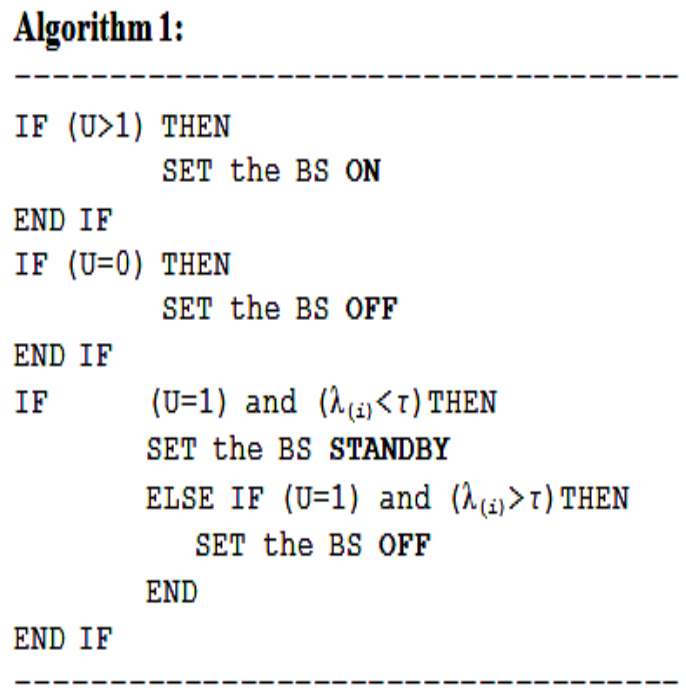

(a) Proposed Algorithm

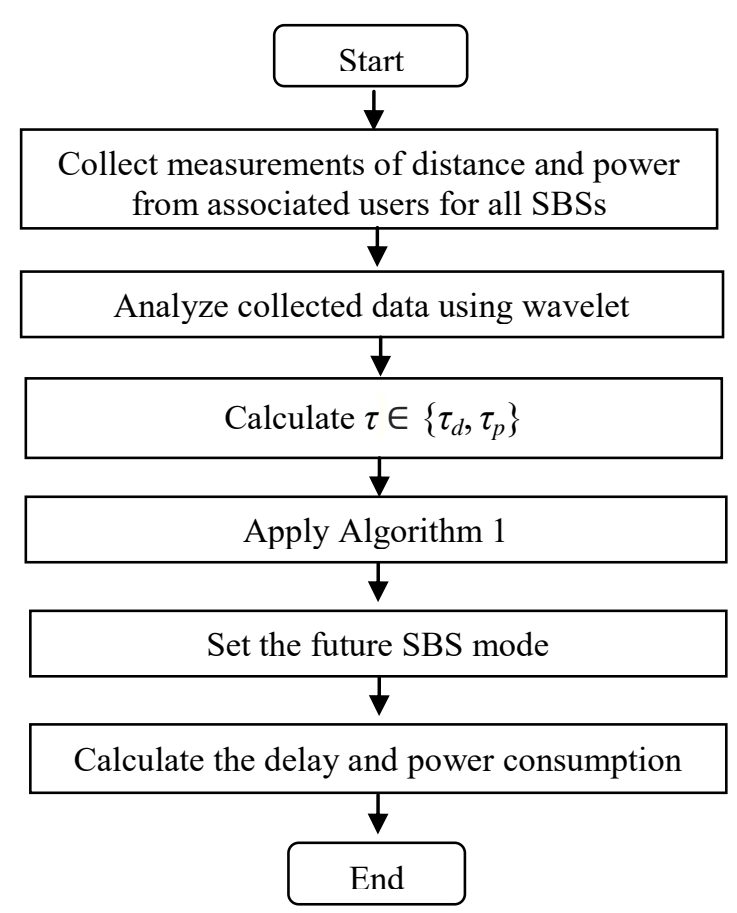

(b) Flowchart of the sleeping mechanism

Fig. 2: Algorithm and flowchart of the proposed sleeping mechanism.

Table II: System Parameters

\begin{tabular}{|l|l|}
\hline \multicolumn{1}{|c|}{ Parameter } & \multicolumn{1}{c|}{ Value } \\
\hline Number of SBSs & 10 \\
\hline Number of users & $1-20$ \\
\hline Carrier frequency $\left(f_{c}\right)$ & $2.4 \mathrm{GHz}$ \\
\hline Shadow fading & Normal distribution, $\mu=0 \mathrm{~dB}, \sigma=4 \mathrm{~dB}$ \\
\hline SBS maximum power & $24 \mathrm{dBm}$ \\
\hline Minimum received power & $-110 \mathrm{dBm}$ \\
\hline
\end{tabular}

\section{Results and Discussion}

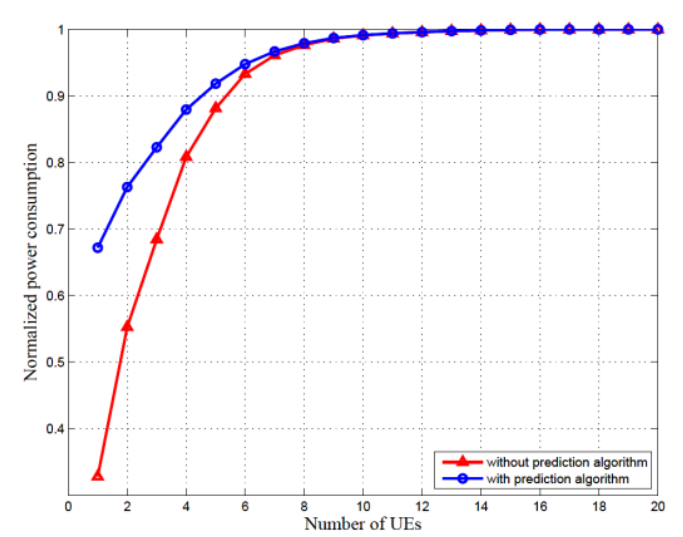

(a)

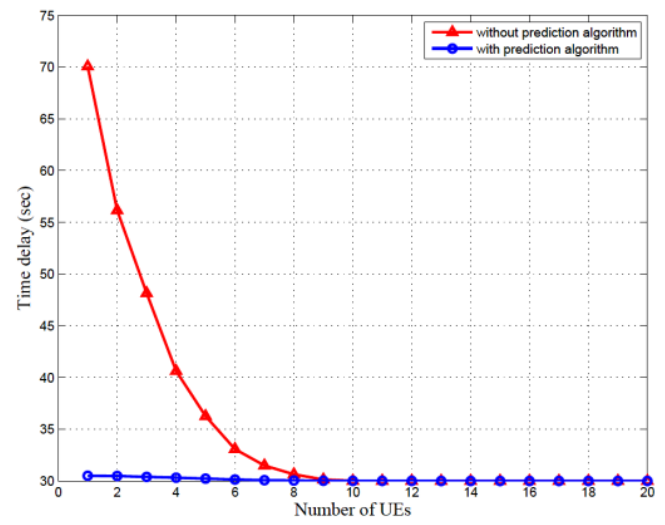

(b)

Fig. 3: (a) Time delay and (b) power consumption of SBS activation based on distance analysis.

In this section, the proposed algorithm is tested, and performance comparisons regarding the time delay and power consumption with respect to the number of user equipments (UEs), with and without the traffic 
prediction algorithm, are obtained. It is worth mentioning that "without prediction" refers to the implementation of Algorithm 1 with the elimination of the conditions $\left(\lambda_{(i)}<\tau\right)$ and $\left(\lambda_{(i)}>\tau\right)$, and when $(U=0$ or 1) the BS enters the Off mode directly. Fig. 3(a) shows the average time delay of SBSs with respect to the number of UEs. It can be noticed from the figure that the proposed algorithm performed well in minimizing the total time delay regardless of the number of associated UEs. The values of delay without the proposed algorithm are approximately double that of the proposed algorithm especially with less number of UEs. When the number of UEs is increased, the delay will decrease until both curves coincide when 9 UEs are associated with the SBS.

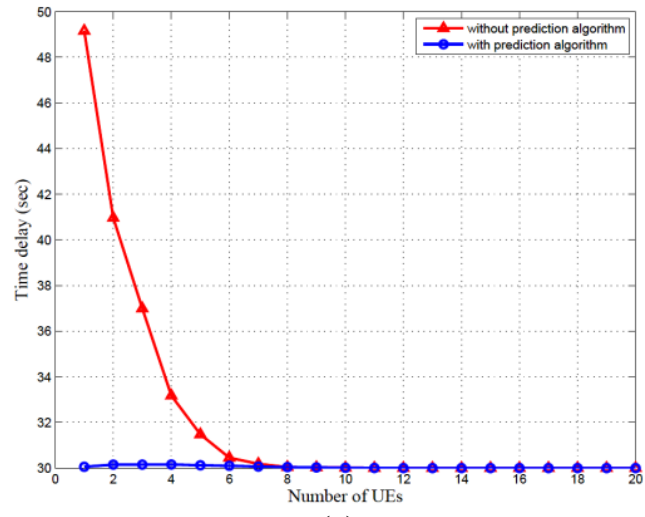

(a)

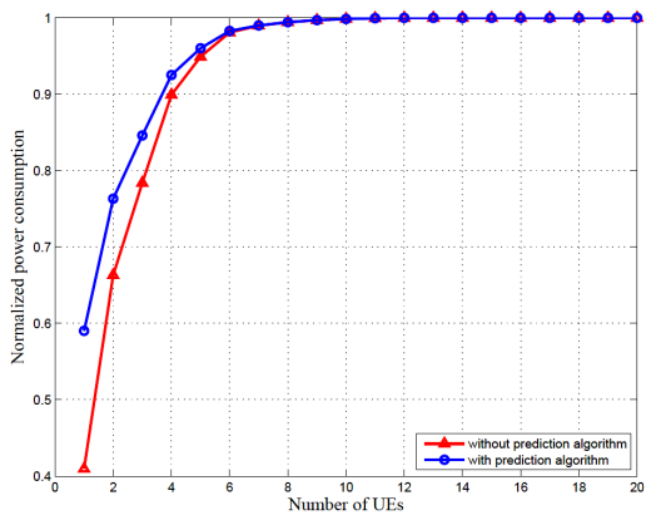

(b)

Fig. 4: (a) Time delay and (b) power consumption of SBS activation based on received power analysis.

A comparison of the normalized power consumption in the network is depicted in Fig. 3(b). It can be observed that the power requirement in the network is higher when the prediction algorithm is implemented, and the power consumption keeps increasing as the number of UEs is increased. The two curves coincide at the points where the number of UEs is 9 or more. This is due to the fact that SBSs with this number of users are set to the On or Standby modes more often than the Off mode in order to minimize the overall time delay experienced by users. Similar comparisons were conducted in Fig. 4; however, the analysis involves the measurements of the received signal power from associated users instead of measured distances.

\section{Conclusion}

In this work, an adaptive sleeping mechanism has been proposed to reduce the overall time delay incurred by SBS activation. The proposed algorithm adaptively modifies the activation of SBSs in a particular cluster through exchanging information among SBSs in that cluster, such that cells are activated prior to receiving a service request. First, the distance measurements between users and SBSs have been adopted for traffic load prediction. Then, the received signal power of associated users was utilized in a second test. Results show that the algorithm significantly reduced the time delay of the SBS sleeping mechanism; however, the power consumption was slightly increased because SBSs are forced to enter the Standby or On states more than the Off state.

\section{References}

[1] S. Song, Y. Chang, X. Wang, H. Xu, T. Sun, and D.Yang. A base Station On-Off Model for Coverage, Throughput and Economy Analysis under HetNet. IEEE Int. Symposium on Personal, Indoor, and Mobile Radio Communication (PIMRC), Sep. 2014, pp. 1808-1813.

[2] J. Peng, H. Tang, P. Hong, and K. Xue. Stochastic Geometry Analysis of Energy Efficiency in Heterogeneous Network with Sleep Control. IEEE Wireless Com. Letters, vol. 2, no. 6, pp. 615-618, Dec. 2013.

[3] E. Mugume and D. K. C. So. Sleep Mode Mechanisms in Dense Small Cell Networks. Proc. IEEE International Conference on Communications (ICC), Jun. 2015, pp. 192-197.

[4] X. Zhang, S. Zhou, Y. Yan, C. Xing, and J. Wang. Energy Efficient Sleep Mode Activation Scheme for Small Cell 
Networks. IEEE Conference on Vehicular Technology (VTC), Sep. 2015, pp. 1-4.

[5] J. Kim, W. S. Jeon, and D. G. Jeong. Effect of Base Station Sleeping Ratio on Energy Efficiency in Densely Deployed Femtocell Networks. IEEE Communications Letters, vol. 19, no. 4, pp. 641-644, Apr. 2015.

[6] G. Yu, Q. Chen, and R.Yin. Dual-Threshold Sleep Mode Control Scheme for Small Cells. IET Communications, vol. 8, no. 11, pp. 2008-2016, Jul. 2014.

[7] X. Guo, Z. Niu, S. Zhou, and P. Kumar. Delay-Constrained Energy- Optimal Base Station Sleeping Control. IEEE Journal on Selected Areas in Communications, vol. 34, no. 5, pp. 1073-1085, May 2016.

[8] C. Liu, B. Natarajan, and H. Xia. Small Cell Base Station Sleep Strategies for Energy Efficiency. IEEE Transactions on Vehicular Technology, vol. 65, no. 3, pp. 1652-1661, Mar. 2016.

[9] G. Wang, C. Guo, S. Wang, and C. Feng. A Traffic Prediction Based Sleeping Mechanism with Low Complexity in Femtocell Networks. IEEE International Conference on Communications (ICC) Workshops, Jun. 2013, pp. $560-565$.

[10] C. Jia and T. J. Lim. Resource Partitioning and User Association with Sleep-Mode Base Stations in Heterogeneous Cellular Networks. IEEE Transaction on Wireless Communications, vol. 14, no. 7, pp. 3780-3793, Jul. 2015.

[11] L.Saker, S. Elayoubi, R. Combes, and T. Chahed. Optimal Control of Wake Up Mechanisms of Femtocells in Heterogeneous Networks. IEEE Journal on Selected Areas in Communications, vol. 30, no. 3, pp. 664-672, Apr. 2012. 